\title{
Selected Experimental Tests of Materials Used in Rapid Prototyping Area
}

Ludmila Novakova-Marcincinova, Jozef Novak-Marcincin, Jozef Torok, Jozef Barna

Faculty of Manufacturing Technologies, Technical University of Kosice, Bayerova 1, 08001 Presov, Slovak Republic

Introduction into problems - Rapid Prototyping (RP) presents the automatic production of physical parts using by additive manufacturing technology. The start techniques for Rapid Prototyping became available in the late 1980s and were used to produce models and prototype parts. Today they are used for a much wider range of applications and are even used to manufacture production-quality parts in relatively small numbers. Rapid Prototyping is widely used in the automotive, aerospace, medical, and consumer products industries. In this paper are presented selected experimental test of materials used in different Rapid Prototyping Technologies. Main part of the paper is oriented on presentation of materials test of Fused Deposition Technology realized by authors on Department of Manufacturing Technology of Faculty of Manufacturing Technology in Presov (Slovakia).

Key words: Rapid Prototyping, Fused Deposition Modelling, materials, experimental testing

\section{Acknowledgements}

Ministry of Education, Science, Research and Sport of SR supported this work, contract VEGA No. 1/0032/12, KEGA No. 002TUKE-4/2012 and ITMS project 26220220125.

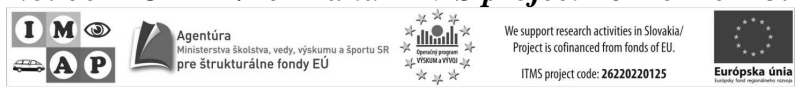

\section{References}

[1] NOVAK-MARCINCIN, J., BARNA, J., NOVAKOVA-MARCINCINOVA, L., FECOVA, V. (2011). Analyses and Solutions on Technical and Economical Aspects of Rapid Prototyping Technology. Tehnicki Vjesnik - Technical Gazette, Vol. 18, No. 4, p. 657-661, ISSN 1330-3651.

[2] NOVAKOVA-MARCINCINOVA, L., BARNA, J., FECOVA, V., JANAK, M., NOVAK-MARCINCIN, J. (2011). Intelligent design of experimental gearbox with rapid prototyping technology support. INES 2011, 15th IEEE International Conference on Intelligent Engineering Systems, Poprad, p. 77-80.

[3] STANEK, M., MANAS, M., MANAS, D., PATA (2011). Possibilities of Rapid Prototyping Technology Using for Design of Plastic Parts. Mechanical Technology, Vol. 16, No. 5, 2011, p. 54-58, ISSN 1211-4162.

[4] SANDA, S., MANAS, M., MANAS, D., STANEK, M., KNOT, J. (2011). Use of Rapid Prototyping in Injection Moulds Design. Mechanical Technology, Vol. 16, No. 5, p. 58-63, ISSN 1211-4162.

[5] GASPAR, S., MASCENIK, J., PASKO, J. (2012). The effect of degassing pressure casting molds on the quality of pressure casting. Advanced Materials Research, No. 428, p. 43-46, ISSN 1022-6680.

[6] GALETA, T., KLJAJIN, M., KARAKASIC, M. (2008). Geometric accuracy by 2D printing model. Strojniski Vestnik/Journal of Mechanical Engineering, Vol. 54, No. 10, p. 725-733.

[7] PILIPOVIC, A., RAOS, P., SERCER, M. (2009). Experimental analysis of properties of materials for rapid prototyping. International Journal of Advanced Manufacturing Technology, Vol. 40, No. 1-2, p. 105-115.

[8] PILIPOVIC, A., RAOS, P., SERCER, M. (2011). Experimental testing of quality of polymer parts produced by Laminated Object Manufacturing - LOM. Tehnicki Vjesnik, Vol. 18, No. 2, p. 253-260.

[9] GALETA, T., RAOS, P., SOMOLANJI, M. (2012). Impact of structure and building orientation on strength of 3D printed models. KGK Kautschuk Gummi Kunststoffe, Vol. 65, No. 10, p. 36-42.

[10] STEFANIC, I., RAOS, P., SAMARDZIC, I., TINTOR, B., MUSSER, E. (2012). Rapid prototyping of casting cores. Tehnicki Vjesnik, Vol. 19, No. 2, p. 459-464.

[11] RAGAN, E., DOBRANSKY, J., BARON, P., KOCISKO, M., SVETLIK, J. (2012). Dynamic of taking out molding parts at injection molding. Metalurgija, Vol. 51, No. 4, p. 567-570, ISSN 0543-5846.

[12] PACURAR, R., BALC, N., PREM, F. (2011). Research on how to improve the accuracy of the SLM metallic parts. In: 14th International Conference on Material Forming Esaform, Belfast, Book Series: AIP Conference Proceedings, Vol. 1353, 2011, p. 1385-1390. 
[13] PACURAR, R., BALC, N., BERCE, P., (2009). Research on Improving the Mechanical Properties of the SLS Metal Parts. In: Annals of DAAAM for 2008 \& Proceedings of the 19th International DAAAM Symposium. Trnava, p. 1003-1004.

[14] AHN, S.-H., MONTERO, M., ODELL, D., ROUNDY, S., WRIGHT, P. K., (2002). Anisotropic material properties of fused deposition modeling ABS. Rapid Prototyping, Vol. 8, No. 4, p. 248-257

[15] NOVAK-MARCINCIN, J., NOVAKOVA-MARCINCINOVA, L., BARNA, J., JANAK, M. (2012). Application of FDM rapid prototyping technology in experimental gearbox development process. Tehnicki Vjesnik, Vol. 19, No. 3, p. 689-694, ISSN 1330-3651.

[16] NOVAK-MARCINCIN, J., JANAK, M., NOVAKOVA-MARCINCINOVA, L. (2012), Increasing of product quality produced by rapid prototyping technology. Manufacturing Technology, Vol. 12, No. 12, p. 71-75, ISSN 1213-2489.

[17] NOVAKOVA-MARCINCINOVA, L., JANAK, M. (2012) Application of progressive materials for RP technology. Manufacturing Technology, Vol. 12, No. 12, p. 76-79, ISSN 1213-2489.

[18] NOVAKOVA-MARCINCINOVA, L., FECOVA, V., NOVAK-MARCINCIN, J., JANAK, M., BARNA, J. (2012). Effective Utilization of RP Technology. Materials Science Forum, Vol. 713, p. 61-66, ISSN 1662-9752.

[19] NOVAKOVA-MARCINCINOVA, L., FECOVA, V., NOVAK-MARCINCIN, J., JANAK, M., BARNA, J. (2012). Effective Utilization of Rapid Prototyping Technology. AIP Conference Proceedings, Vol. 1431, p. 834841, ISSN 0094-243X.

[20] VALICEK J., HLOCH S., KOZAK D. (2009). Surface geometric parameters proposal for the advanced control of abrasive waterjet technology. International Journal of Advanced Manufacturing Technology, Vol. 41, No. 3-4, p. 323-328, ISSN 0268-3768.

[21] KOVACEVIC, D., BUDAK, I., ANTIC, A., KOSEC, B. (2011). Special Finite Elements: Theoretical background and Application, Tehnicki Vjesnik, Vol. 18, No. 4, p. 649-655, ISSN 1330-3651.

[22] MURCINKO, J., MURCINKOVA, Z. (2012). On-line monitoring system applied to explosive conditions of printing machine dryers. WIT Transactions on Information and Communication Technologies, Vol 44, p. 305-316, ISSN 1743-3517.

[23] TIC, V., LOVREC, D. (2012). Design of modern hydraulic tank using fluid flow simulation. International Journal of Simulation Modelling, Vol. 11, No. 2, p. 77-88, ISSN 1726-4529.

[24] MICHALIK, P., ZAJAC, J., DUPLAK, J., PIVOVARNIK, A. (2012). CAM software products for creation of programs for CNC machining. Lecture Notes in Electrical Engineering, Vol. 1, No. 141, p. 421-425, ISSN 18761100 .

[25] NOVAKOVA-MARCINCINOVA, L., NOVAK-MARCINCIN, J., BARNA, J., TOROK, J. (2012). Special materials used in FDM rapid prototyping technology application. INES 2012 - IEEE 16th International Conference on Intelligent Engineering Systems, Proceedings, art. no. 6249805, p. 73-76. 